\title{
Reconfigurable Plasmonic Diastereomers Assembled by DNA
}

\section{Origami}

Meng Wang, ${ }^{\dagger},+, \xi$ Jinyi Dong, ${ }^{\dagger, \xi}$ Chao Zhou, ${ }^{*}$, Hao Xie,${ }^{\S}$ Weihai Ni, ${ }^{\S}$ Shun Wang, ${ }^{\dagger}$ Huile Jin, ${ }^{* \dagger}$ Qiangbin Wang*,*

†nstitute of New Materials and Industrial Technologies, Wenzhou University, Wenzhou 325035, P. R. China

$\ddagger$ CAS Key Laboratory of Nano-Bio Interface, Division of Nanobiomedicine and $i$-Lab, Suzhou Institute of Nano-Tech and Nano-Bionics, Chinese Academy of Sciences, Suzhou 215123, P. R. China

§Jiangsu Key Laboratory of Thin Films, School of Physical Science and Technology, Soochow University, Suzhou 215006, P. R. China

$\xi$ These authors contribute equally to this work.

Corresponding Authors

*E-mail: czhou2018@sinano.ac.cn

*E-mail: huilejin@wzu.edu.cn

*E-mail: qbwang2008@sinano.ac.cn

\section{Materials}

All the chemicals were commercially obtained. Tetra chloroauric acid $\left(\mathrm{HAuCl}_{4} \cdot 4 \mathrm{H}_{2} \mathrm{O}\right)$, potassium bromide $(\mathrm{KBr})$, silver nitrate $\left(\mathrm{AgNO}_{3}\right)$, tris(2-carboxyethyl) phosphine (TCEP) was purchased from Alfa Aesar, and cetyltrimethylammonium bromide (CTAB), sodium dodecyl sulfate (SDS), sodium borohydride $\left(\mathrm{NaBH}_{4}\right)$, ascorbic acid was supplied by Sigma Aldrich.

Non-thiolated DNA sequences were bought from GENEWIZ Suzhou, China. Thiolated 
DNA sequences of HPLC grade were bought from Sangon Biotech (shanghai) Co.,ltd.

The p8064 DNA scaffold DNA was purchased from tilibit nanosystems ${ }^{\circledR} \mathrm{GmbH}$ (Garching, Germany).

\section{DNA origami template}

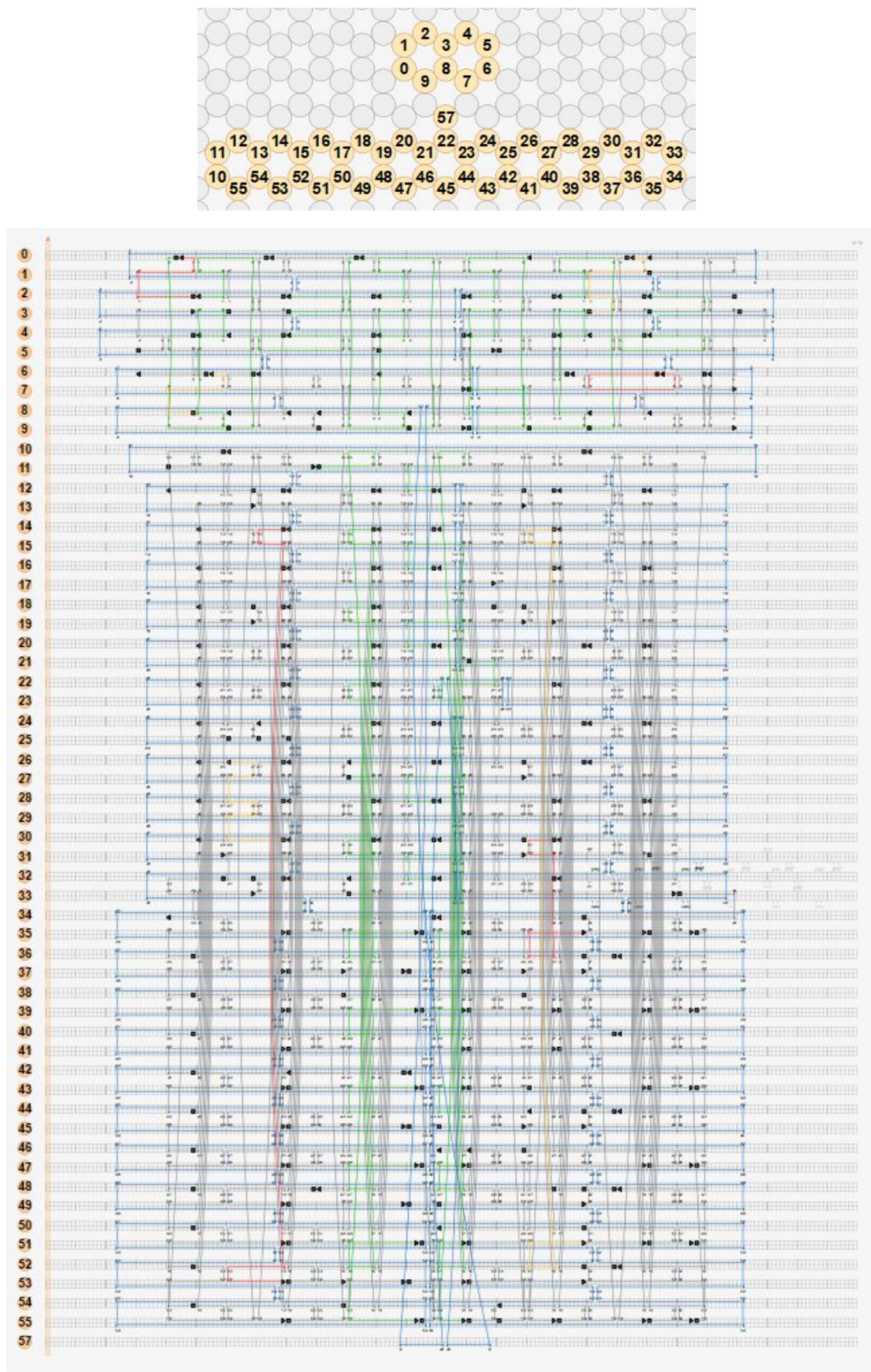

Figure S1. Routing scheme of the DNA origami template designed by caDNAno software. 


\section{Synthesis of the AuNRs.}

The seed solution for gold NRs was prepared firstly. A $50 \mu \mathrm{L}$ amount of $50 \mathrm{mM} \mathrm{HAuCl}_{4}$ was mixed with $10 \mathrm{~mL}$ of $0.1 \mathrm{M} \mathrm{CTAB}$ solution. A $0.6 \mathrm{~mL}$ portion of fresh cold 0.01 $\mathrm{M} \mathrm{NaBH}_{4}$ was diluted to $1 \mathrm{~mL}$ with water and was then injected into the $\mathrm{Au}$ (III) CTAB solution under vigorous stirring (1200 rpm). The solution color changed from yellow to brownish-yellow, and the stirring was stopped after $2 \mathrm{~min}$. The seed solution was aged at $25^{\circ} \mathrm{C}$ for $30 \mathrm{~min}$ before use. To prepare the growth solution, $3.6 \mathrm{~g}$ of CTAB together with $1.4 \mathrm{~g} \mathrm{KBr}$ were dissolved in $100 \mathrm{~mL}$ of warm water $\left(50-70{ }^{\circ} \mathrm{C}\right)$. The solution was allowed to cool to $30{ }^{\circ} \mathrm{C}$, when a $2.4 \mathrm{~mL}$ of $4 \mathrm{mM} \mathrm{AgNO}$ s solution was added. The mixture was kept undisturbed at $30^{\circ} \mathrm{C}$ for $15 \mathrm{~min}$, after which $50 \mathrm{~mL}$ of 1 $\mathrm{mM} \mathrm{HAuCl}_{4}$ solution was added. After $15 \mathrm{~min}$ of slow stirring $(400 \mathrm{rpm}), 0.9 \mathrm{~mL}$ of 64 $\mathrm{mM}$ ascorbic acid was added, and the solution was vigorously stirred for $30 \mathrm{~s}$ until it became colorless. Finally, $0.16 \mathrm{~mL}$ of seed solution was injected into the growth solution. The resultant mixture was stirred for $30 \mathrm{~s}$ and left undisturbed at $30{ }^{\circ} \mathrm{C}$ for 12 $\mathrm{h}$ for AuNR growth. The reaction products were isolated by centrifugation at $8000 \mathrm{~g}$ for 30min followed by removal of the supernatant and the precipitates were re-dispersed in water.

\section{Functionalization of the AuNRs}

Thiolated DNA strands were incubated with TCEP [tris(2-carboxyethyl) phosphine] for at least $1 \mathrm{~h}$ to reduce the disulfide bonds. The ration of DNA:TCEP was 1:200. Before functionalization, the AuNRs were spun down and then resuspended with ultrapure water (18.2 M $\Omega . \mathrm{cm})$ to remove excess cetyl trimethylammonium bromide (CTAB). 
AuNRs $(1 \mathrm{nM}, 500 \mu \mathrm{L})$ were mixed with $\mathrm{H}_{2} \mathrm{O}(150 \mu \mathrm{L}), 0.2 \%$ sodium dodecyl sulfate (SDS, $100 \mu \mathrm{L}), 10 \times \mathrm{TBE}(50 \mu \mathrm{L}), 1 \mathrm{M} \mathrm{HCl}(50 \mu \mathrm{L}, \mathrm{pH}=3)$ and $250 \mathrm{mM}$ thiolated DNA strands $(10 \mu \mathrm{L}) . \mathrm{NaCl}(5 \mathrm{M}, 10 \mu \mathrm{L})$ was added every 20 min for 9 times. Subsequently, $1 \mathrm{M} \mathrm{NaOH}(50 \mu \mathrm{L})$ was added to adjust the $\mathrm{pH}$ value back to 8 . Finally, the concentration of $\mathrm{NaCl}$ reached $500 \mathrm{mM}$. The AuNRs functionalized with DNA were purified to remove excess free DNA strands by centrifugation before use.

\section{Gel purification and recycle of the self-assembled AuNR nanostructures}

Under visible light, the target bands are cut down carefully, and placed it on a flat plate wrapped with parafilm, then the gels are cut into small pieces. With a glass slide wrapped with parafilm, the small gel pieces are carefully squeezed to generate solutions. At last the squeezed solution are collected (avoid collecting gel particles) as sample solution.

\section{Characterization}

The absorption spectrum of the DNA origami templates and the AuNRs were measured by using Thermo Scientific UV-vis Spectrometer. The CD spectrum were collected on an Applied Photophysics Chirascan Plus Spectrometer. A Tecnai G2 F20 S-Twin TEM (200 kv) and Hitachi HT7700 TEM (100 kv) were used for sample observation.

\section{The adding order of fuel strands to reconfigure the plasmonic diastereomers.}

We first assembled the sample with all blocking strands. At this state, all chiral centers were at a random state (labeled as $\mathrm{O}$ ). To minimize the volume change during addition of fuel strands, we divided the sample into two identical aliquots and treated them in parallel. Then we added fuel strands as depicted in the following table (Table S1). It 
should be noted that only states without random chiral centers (marked by *) were presented in Figure 4.

Table S1. The adding order of fuel strands to reconfigure the plasmonic diastereomers. The left-handed, right-handed, relaxed state are abbreviated as L, R, and O. The marked * mean the conformation listed in the main text.

\begin{tabular}{|c|c|c|c|c|c|}
\hline Order & Fuel strands & Conformation & Order & Fuel strands & Conformation \\
\hline 1 & $\mathrm{~L}_{1}$-activator $0.2 \mu \mathrm{L}$ & $\mathrm{L}-\mathrm{O}-\mathrm{O}$ & 1 ' & $\mathrm{R}_{1}$-activator $0.2 \mu \mathrm{L}$ & $\mathrm{R}-\mathrm{O}-\mathrm{O}$ \\
\hline 2 & $\mathrm{~L}_{2}$-activator $0.2 \mu \mathrm{L}$ & L-L-O & 2 ' & $\mathrm{R}_{2}$-activator $0.2 \mu \mathrm{L}$ & R-R-O \\
\hline 3 & $\mathrm{~L}_{3}$-activator $0.2 \mu \mathrm{L}$ & L-L-L* & $3^{\prime}$ & $\mathrm{R}_{3}$-activator $0.2 \mu \mathrm{L}$ & $\mathrm{R}-\mathrm{R}-\mathrm{R}^{*}$ \\
\hline 4 & $\mathrm{~L}_{1}$-blocker $0.4 \mu \mathrm{L}$ & O-L-L & $4^{\prime}$ & $\mathrm{R}_{1}$-blocker $0.4 \mu \mathrm{L}$ & O-R-R \\
\hline 5 & $\mathrm{R}_{1}$-activator $0.2 \mu \mathrm{L}$ & R-L-L* & 5, & $\mathrm{~L}_{1}$-activator $0.2 \mu \mathrm{L}$ & L-R-R* \\
\hline 6 & $\mathrm{~L}_{2}$-blocker $0.4 \mu \mathrm{L}$ & $\mathrm{R}-\mathrm{O}-\mathrm{L}$ & 6 & $\mathrm{R}_{2}$-blocker $0.4 \mu \mathrm{L}$ & L-O-R \\
\hline 7 & $\mathrm{R}_{2}$-activator $0.2 \mu \mathrm{L}$ & R-R-L* & 7 & $\mathrm{~L}_{2}$-activator $0.2 \mu \mathrm{L}$ & L-L-R* \\
\hline 8 & $\mathrm{~L}_{3}$-blocker $0.4 \mu \mathrm{L}$ & $\mathrm{R}-\mathrm{R}-\mathrm{O}$ & 8, & $\mathrm{R}_{3}$-blocker $0.4 \mu \mathrm{L}$ & L-L-O \\
\hline 9 & $\mathrm{R}_{3}$-activator $0.2 \mu \mathrm{L}$ & R-R-R & $9^{\prime}$ & $\mathrm{L}_{3}$-activator $0.2 \mu \mathrm{L}$ & L-L-L \\
\hline 10 & $\begin{array}{l}\mathrm{R}_{1} \text {-blocker } 0.4 \mu \mathrm{L} \\
\mathrm{R}_{3} \text {-blocker } 0.4 \mu \mathrm{L} \\
\mathrm{L}_{1} \text {-activator } 0.8 \mu \mathrm{L} \\
\mathrm{L}_{3} \text {-activator } 0.8 \mu \mathrm{L}\end{array}$ & L-R-L* & $10^{\prime}$ & $\begin{array}{l}\mathrm{L}_{1} \text {-blocker } 0.4 \mu \mathrm{L} \\
\mathrm{L}_{3} \text {-blocker } 0.4 \mu \mathrm{L} \\
\mathrm{R}_{1} \text {-activator } 0.8 \mu \mathrm{L} \\
\mathrm{R}_{3} \text {-activator } 0.8 \mu \mathrm{L}\end{array}$ & R-L-R* \\
\hline
\end{tabular}
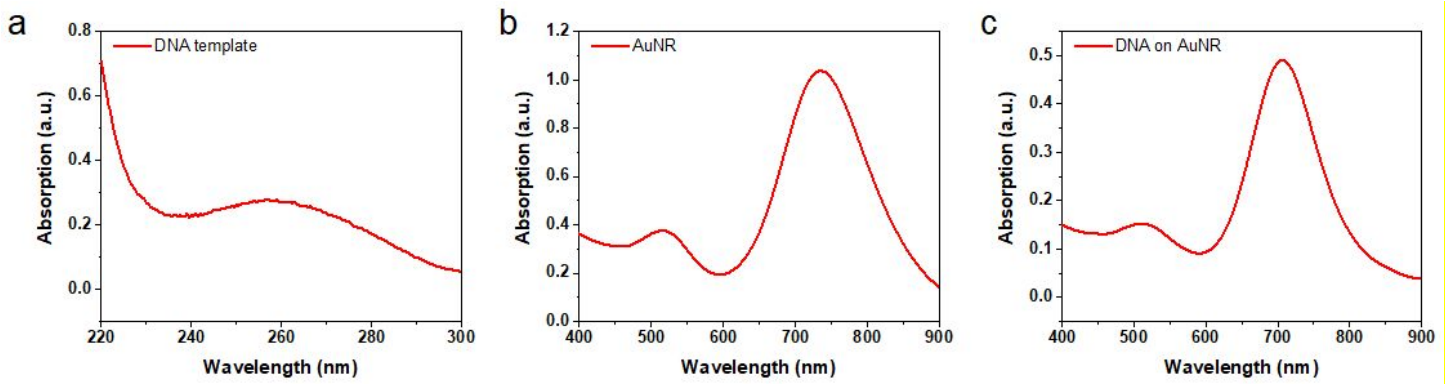

Figure S2. UV-vis spectrum of DNA origami template (a), free AuNRs (b) and DNAmodified AuNRs (c). The resonance peak of AuNRs showed slight blue-shift, suggesting the successful coating of DNA strands. 

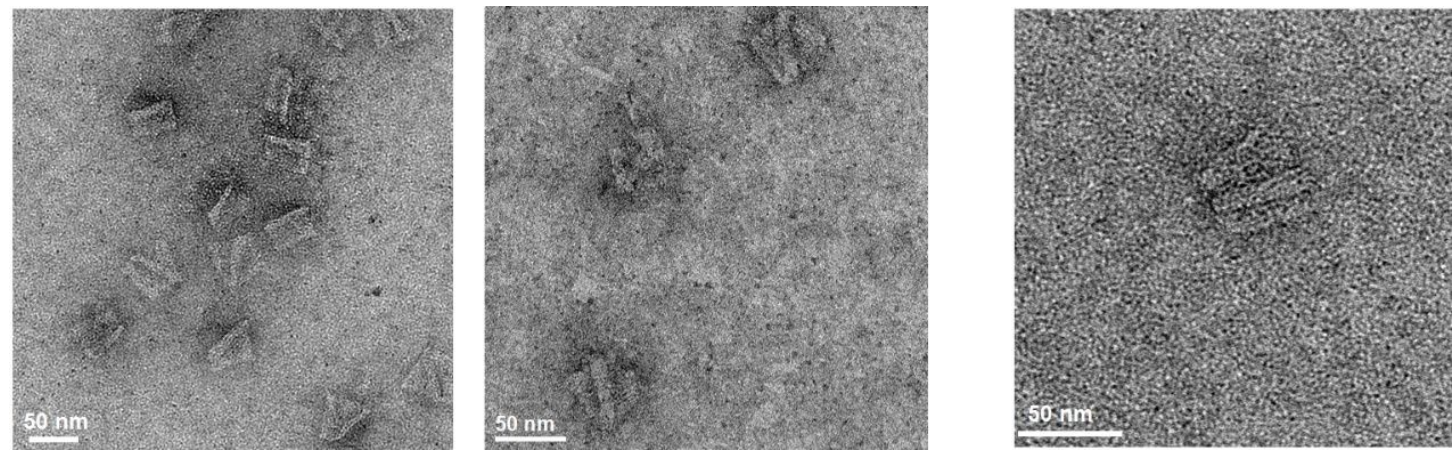

Figure S3. TEM images of DNA origami templates.
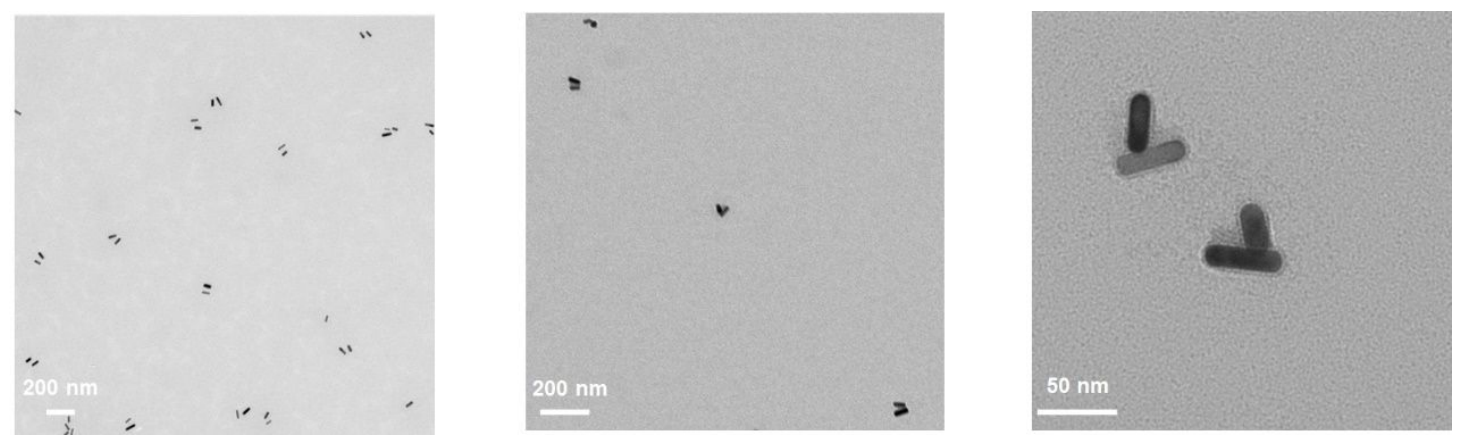

Figure S4. TEM images of AuNR dimers.
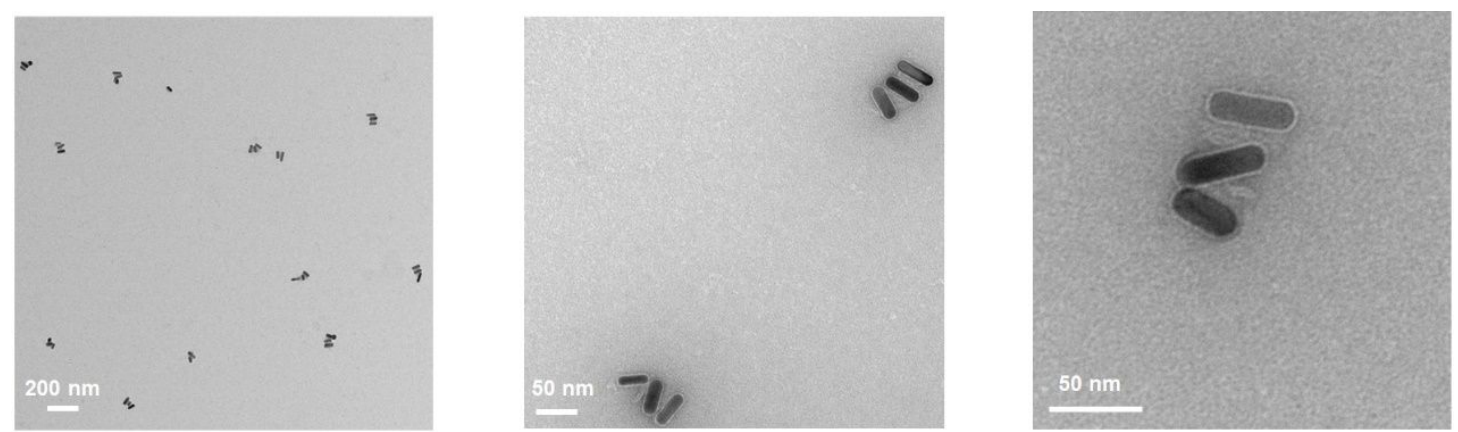

Figure S5. TEM images of AuNR trimers. 

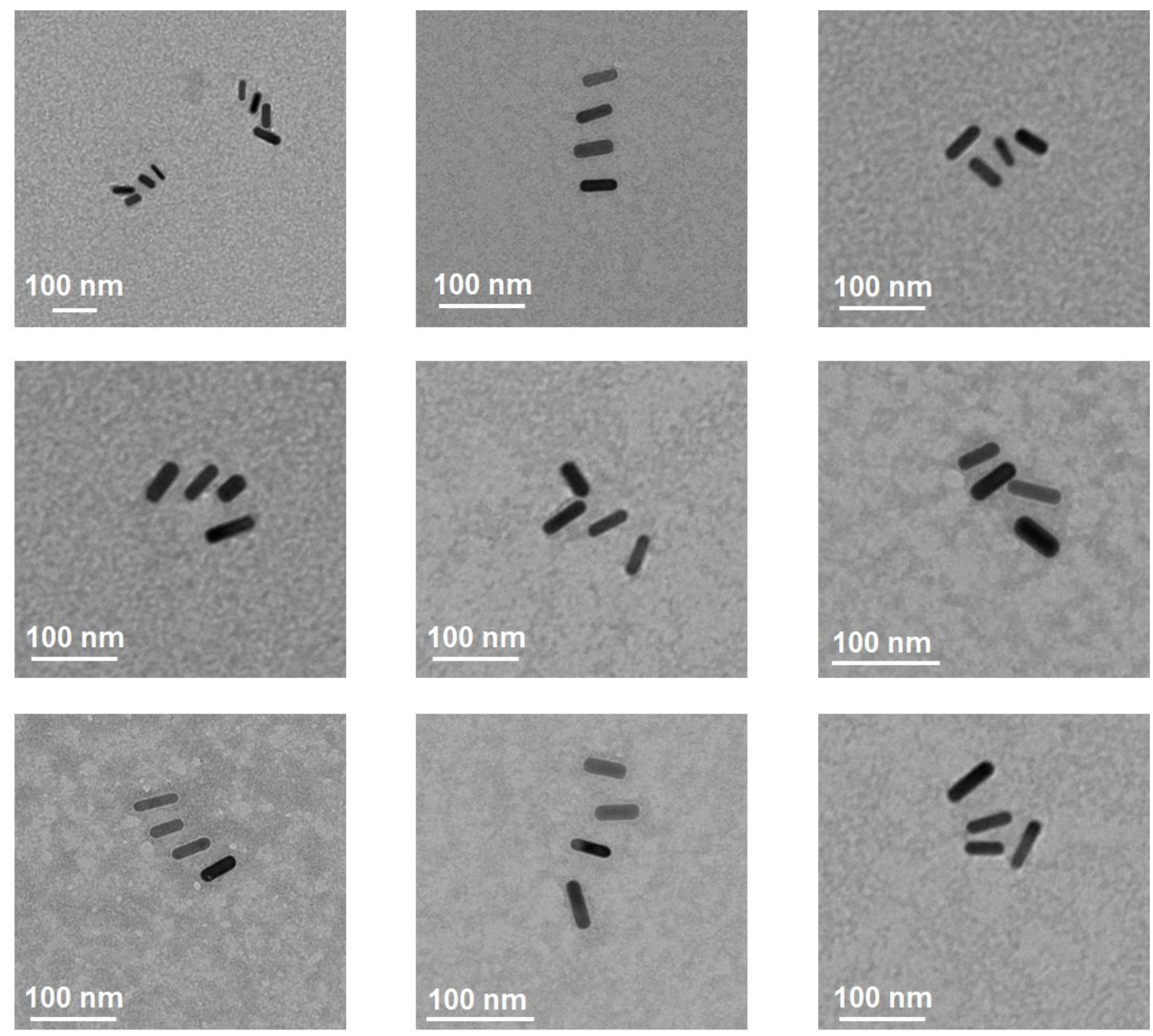

Figure S6. TEM images of AuNR tetramers. 

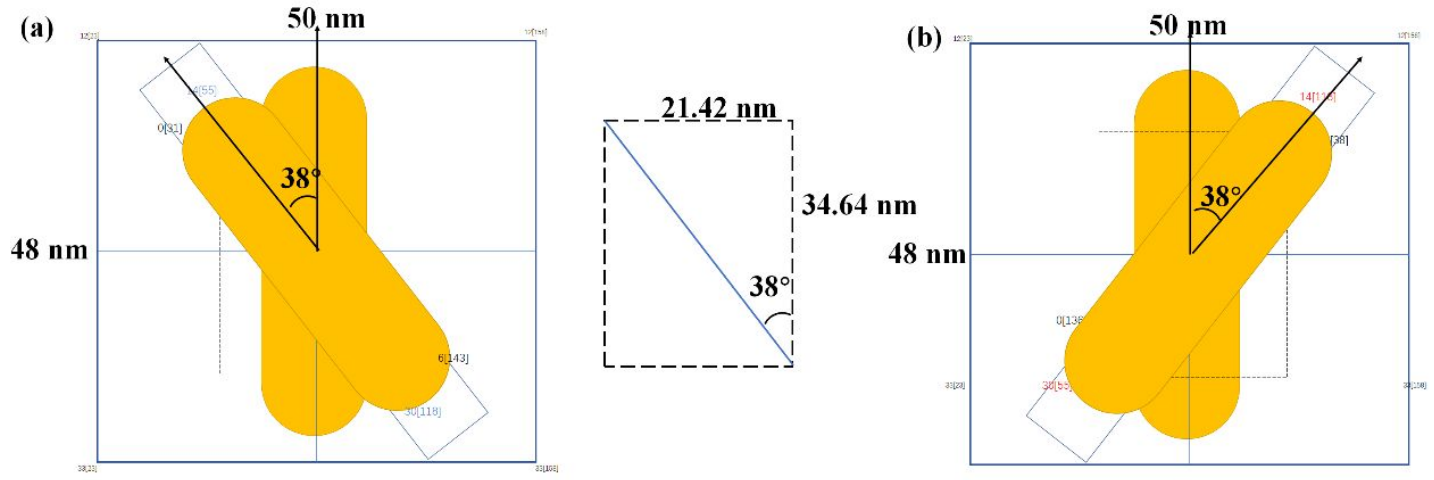

(c)

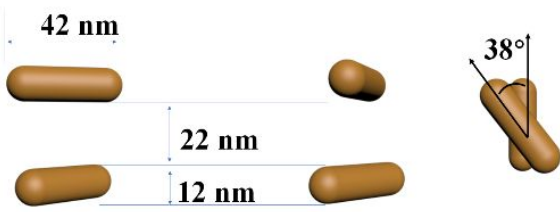

(d)

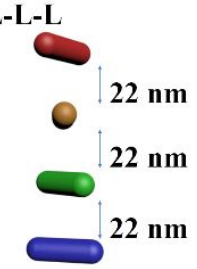

Figure S7. Scheme for calculating parameters in DNA templates and AuNR nanostructures.
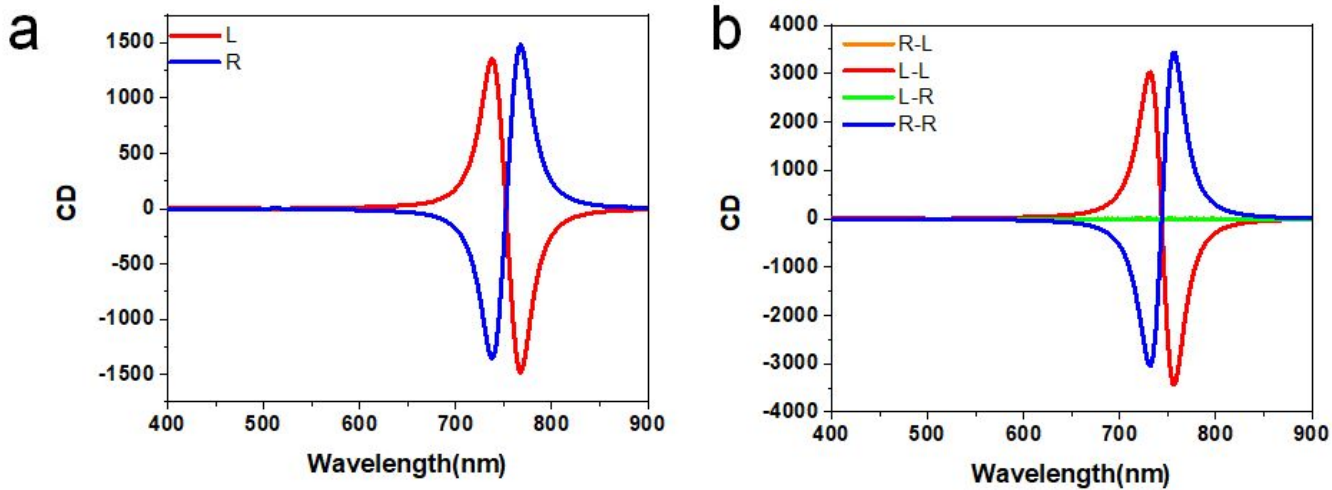

Figure S8. Theoretical calculated CD spectrum of one chiral center of AuNR dimers (a), two chiral centers of AuNR trimers (b). 


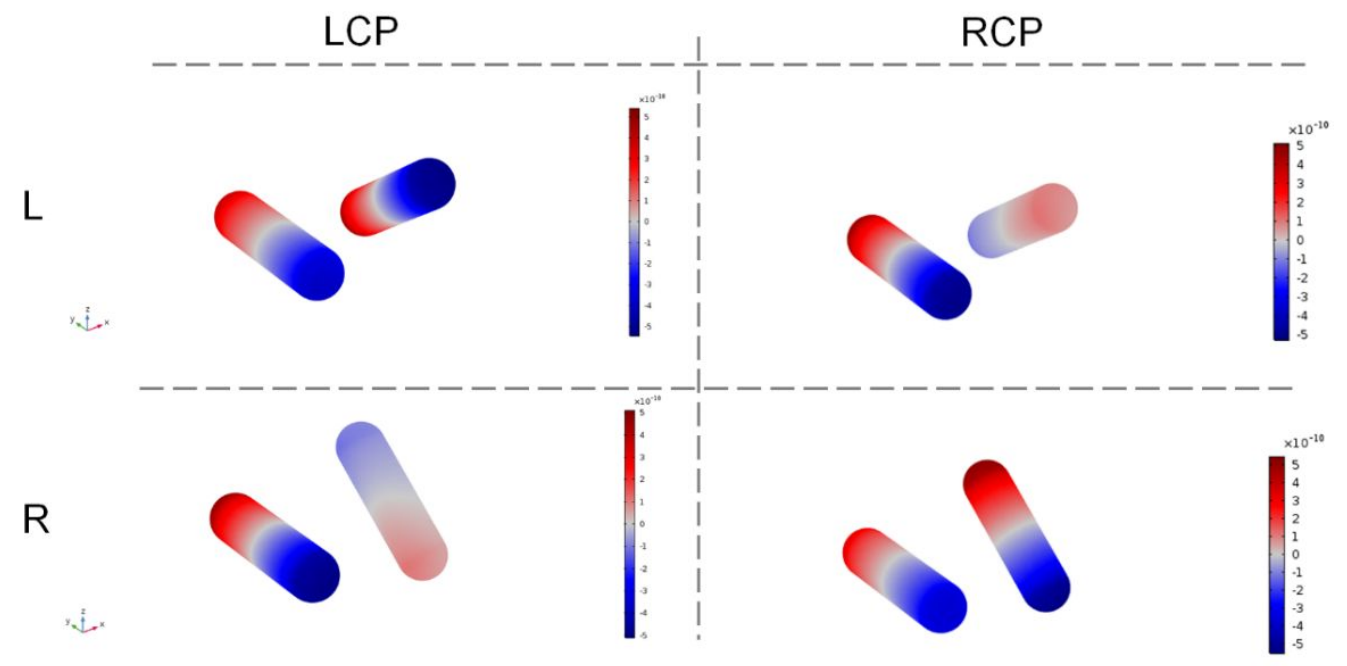

Figure S9. Theoretically calculated AuNR dimers under left and right circularly polarized light (LCP and RCP).

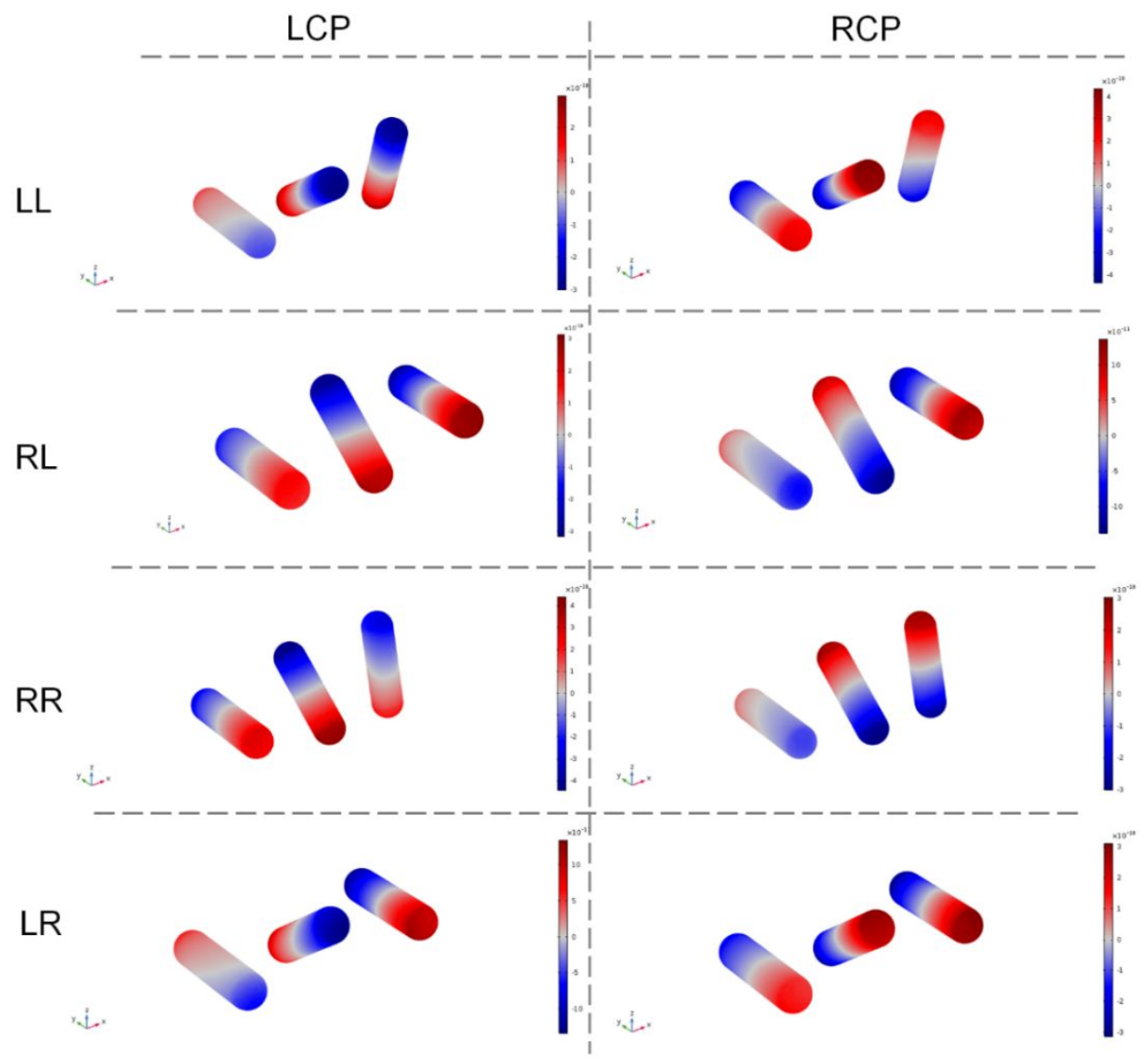

Figure S10. Theoretically calculated AuNR trimers under left and right circularly polarized light (LCP and RCP). 


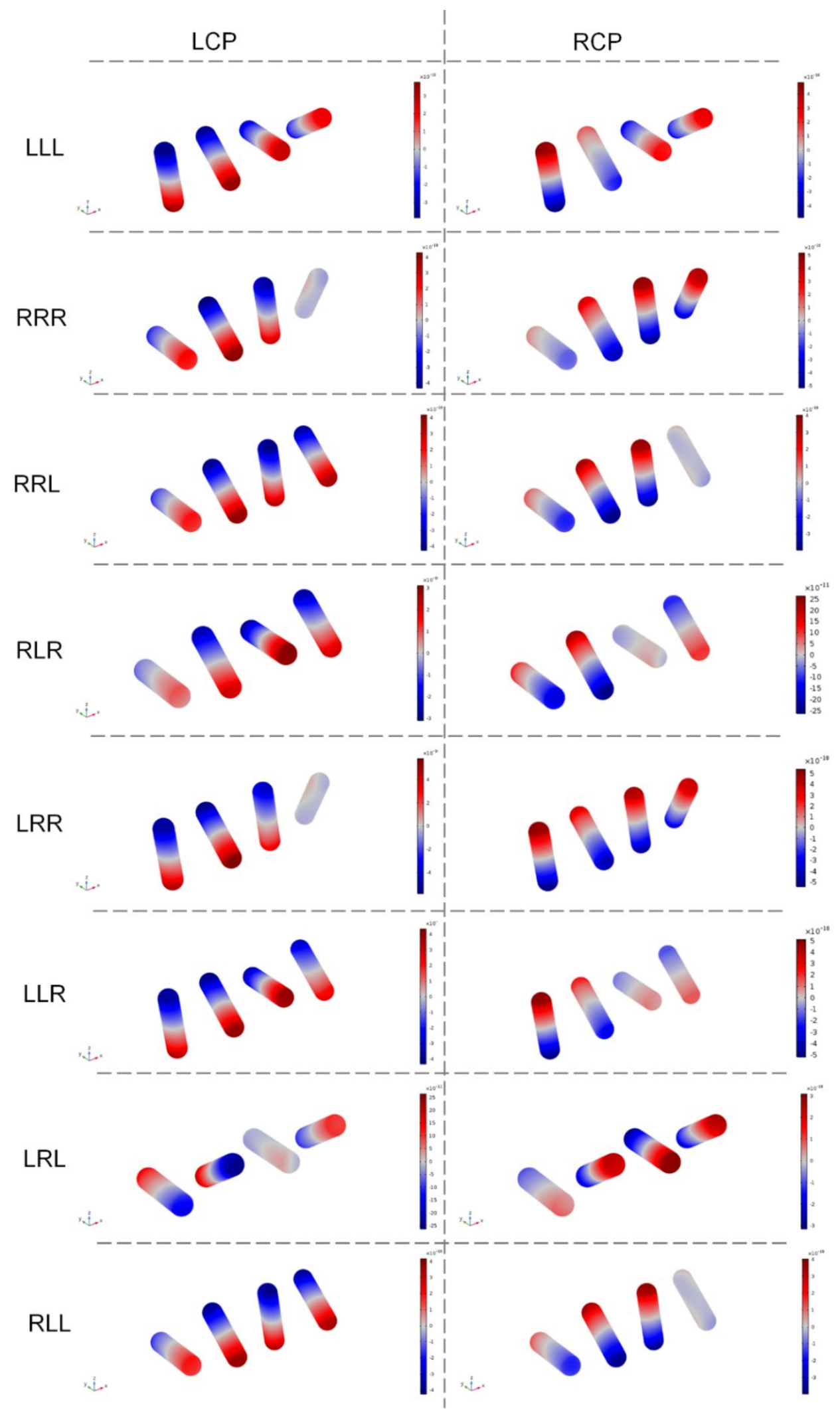

Figure S11. Theoretically calculated AuNR tetramers under left and right circularly polarized light (LCP and RCP). 
DNA Sequences.

Table S2. Staple strands of DNA origami Plate

\begin{tabular}{|c|c|c|c|}
\hline Start & End & Sequences 5'-3' & Length \\
\hline 12[139] & $10[126]$ & AGTTGAGAAACGAAATTAAGAGGCTGCACGTATAAAACACTA & 42 \\
\hline $12[55]$ & $10[42]$ & СCTCAAATTACCCTGTGAATTTGTGAGAAAACAGCGGTAATA & 42 \\
\hline $54[69]$ & $11[62]$ & CATAAAATTTCTGCAAAAGAAGTTTTGCTCAGCCA & 35 \\
\hline $55[119]$ & $12[119]$ & CAGTGCCTACATGGGTCCAGACGACGTAATGCATTTTCGAGC & 42 \\
\hline $55[151]$ & $12[140]$ & CTAATTACGAGGCATAGCCACATTATTCATC & 31 \\
\hline $10[41]$ & $12[28]$ & GTAAAATTCGTCATAACAGTTCAGAAAA & 28 \\
\hline $55[56]$ & $12[56]$ & ACTTAAAAAATCCCGAATAAACACCGGATTACCAGTATAACC & 42 \\
\hline $55[87]$ & $12[77]$ & TTAAAAAATAGCGAGAGGGGCTTAACGTTATA & 32 \\
\hline $55[105]$ & 12[91] & AGTGCCTTACCAGACGACGATATTTAACTATAAAGTATAGTA & 42 \\
\hline $16[118]$ & $13[111]$ & CATCCTAATTTACGCAACAATGCTAATGGGTAAAG & 35 \\
\hline $16[55]$ & $13[48]$ & GTTTTAATTCGAGCAAAGACTTCAGAAGCAGGTCT & 35 \\
\hline $52[125]$ & $14[119]$ & TATTCACTCCTGAACAAGATTAAACAATAAACAAC & 35 \\
\hline $11[28]$ & $14[35]$ & TGCGGAAGTTTAGAAAAAAGAGACGCAGGATAGACCAAAAATCAAAGCG & 49 \\
\hline $53[56]$ & $14[56]$ & AAAAAGCGTTCCGGTCTAGTTAATTTCACGTTAAATAAGACT & 42 \\
\hline $53[84]$ & $14[77]$ & TGAAGGGATGGGCGGTTGTGTTTACTAGTGTGATA & 35 \\
\hline $55[98]$ & $14[91]$ & CGGGGTCGGAGTGTGACAAAACAGAACGCGAAATA & 35 \\
\hline $50[125]$ & $16[119]$ & GAGCCGCTTTCCTTATCATTTATAAAATAATATCC & 35 \\
\hline $52[132]$ & $16[140]$ & CGAGAGAGTAGACTCCTCAAGAGAAGGACGGATAATGGCTCAAATTACC & 49 \\
\hline $54[34]$ & $16[35]$ & TTTCTCCGTACAGCGCCATGTCGGGAACAAGCCCGTTCAAAG & 42 \\
\hline $51[56]$ & $16[56]$ & CGCGGTCGGAACGTACATATAACTATATTCAAATATATTTGC & 42 \\
\hline $51[87]$ & $16[77]$ & GCCAATGGTAAAGTTAAAACCTAAAGCGAGAA & 32 \\
\hline $53[98]$ & $16[91]$ & AGTCTCTTCATTAATGTTTATAGCATGTAGAAGAC & 35 \\
\hline $46[111]$ & $17[104]$ & ATCGGCACATCTTTACCGGAAGCCACCCACCACCACCAGAGCACCAATC & 49 \\
\hline $52[34]$ & $18[35]$ & GGATAACCGACGGCCAGTGCCGGTAACGAACAGGTTGATAAG & 42 \\
\hline $48[62]$ & $18[56]$ & GGCAGCAGCAGAAGAGTCAATTTAGGTTGGGTTCT & 35 \\
\hline 49[84] & $18[77]$ & TCTGGCGAGCTCCGGCCAGAGCTGATGCTTTTAAC & 35 \\
\hline $22[118]$ & $19[111]$ & AACCTCCCGACTAAGCTTATCATAGCAAAGCAAGC & 35 \\
\hline $48[125]$ & $19[118]$ & GAACCAGATTTTCATCGTATGAATCCAAGAACGGGTATTAAACGTTTTT & 49 \\
\hline $25[42]$ & $19[48]$ & AATAGTAATTTTCAAATGGTCTCTGCGATTCCATAACATGTTTTGCTGA & 49 \\
\hline $48[118]$ & $20[126]$ & AGCCACCTCATAATCAAAATCTGTAGCGCTAAGAACGCGACAGCGGAAT & 49 \\
\hline $48[132]$ & $20[140]$ & ATAGGCGCTAACCGCCACCCTCAGAACCGGATAGCAGTGAATATCAACG & 49 \\
\hline $50[34]$ & $20[35]$ & CCAGGGTCTGGCGAAAGGGGGAAGGGCGAGCTTAATTAAATA & 42 \\
\hline $47[56]$ & $20[56]$ & CGGTGGTATTGCAGTGTCTGTAAATCGTATTAAGACGCTGTG & 42 \\
\hline 47[87] & $20[77]$ & GCCCGCTCAGCAGCAACCTTATCAATAGCGAT & 32 \\
\hline 49[91] & $20[91]$ & CCACCCTCAGAACCCCGCCTCGAGAACAGCAAATCAGTCCTT & 42 \\
\hline $44[125]$ & 22[119] & GAAACGTTGAAGCCTTAAAGAATGGCGTTTTAGCG & 35 \\
\hline $48[34]$ & $22[35]$ & ATCGGTGAAAGCGCCATTCGCCAGCTTTAGTTTCAACGAGTA & 42 \\
\hline $45[56]$ & $22[56]$ & TTTCGCAGCTGGTATGGAACAAGAGTCCAATAACCTTGCTAT & 42 \\
\hline $45[84]$ & $22[77]$ & GCGGTAACCTGGAGGTGTCCAAATTAATAATATAT & 35 \\
\hline
\end{tabular}




\begin{tabular}{|c|c|c|c|}
\hline $43[112]$ & $24[126]$ & TTACCATTAGCAAGTCACCGTTAACGAGCGTCTTTTTTCAAG & 42 \\
\hline $44[132]$ & $24[140]$ & TGTATCGAGCCCTCATAGTTAGCGTAACGTAAATGCAGATGACGAACTG & 49 \\
\hline $46[34]$ & $24[35]$ & CCGGCACCGACGACAGTATCGGGGCGCATTTCGCATTTGGGG & 42 \\
\hline $25[56]$ & $24[49]$ & TAGTGGTTCCGAAATGTTCCAGTTTGTTTAGCTAT & 35 \\
\hline $43[87]$ & $24[77]$ & CTCAGTATGAGCCGGGTCAAGAACGATAGGGT & 32 \\
\hline $45[91]$ & $24[91]$ & TCAGTAGCGACAGATCGATAGAAAGGGCAGCTACAATGAATA & 42 \\
\hline $41[119]$ & $26[126]$ & AAGGTGAAAGACAAAATAAGAAACGAGAACTTCCA & 35 \\
\hline $44[34]$ & $26[35]$ & TCGTAACGGATTGACCGTAATAGTAACATTCTACTACAGGCA & 42 \\
\hline $41[56]$ & $26[56]$ & ATGCCGGGTGTCACAGCACCGCCTGGCCTCCTGTTTGATGAC & 42 \\
\hline $42[83]$ & $26[70]$ & TGGTGTGAATCCCTCCAGCAGGCGAAAA & 28 \\
\hline $43[98]$ & $26[91]$ & CACCAGTATTTGGGTATCCTGAAATAAACACGCTG & 35 \\
\hline $38[111]$ & $27[111]$ & GACACCAAAATTCAACATTCAACCGATTCATATTATTTATCC & 42 \\
\hline $39[119]$ & $28[119]$ & TACCAGCAAAAGAAACAGGGAAGCGCAAGATTTTTTGTTTAA & 42 \\
\hline $40[132]$ & $28[140]$ & TCGGTCAATGAATAATAATTTTTTCACGAGCCTTTCATCGCCAACAAAG & 49 \\
\hline $42[34]$ & $28[35]$ & ACCCGTCTTCCTGTAGCCAGCCCAATAGCAATAAAAAAAACA & 42 \\
\hline $39[56]$ & $28[56]$ & CGCCTGTCGGGGGTCAGAGAGGCGGTTTCTGATTGCCCTTCT & 42 \\
\hline $39[87]$ & $28[77]$ & ATTGGGCCCCCTGCATCAAGTTGCAGAGACGG & 32 \\
\hline $30[111]$ & 28[91] & TGAACACAGAATAAAATGAAAATAGCAGCCCTTTT & 35 \\
\hline $36[125]$ & $30[119]$ & AACTGGCATATCAGAGAGAGTAAATTAGACGGGAG & 35 \\
\hline $40[34]$ & $30[35]$ & GAACGCCTTCGCATTAAATTTCAAATATGGAGAAGGAACCCT & 42 \\
\hline $37[56]$ & $30[56]$ & GCCAGCAGTACCGACACGTTGCGCTCACCCAACGCGCGGGAG & 42 \\
\hline $37[84]$ & $30[77]$ & CGTGATATTGCGGCGGGCCGTGGGCGCCCATTAAT & 35 \\
\hline $39[98]$ & $30[91]$ & CAATAGACGGAATATACAGAGCCTGAACAATCGTG & 35 \\
\hline $35[98]$ & $31[111]$ & AGGAAACGAACAAACAAGAAACAATGAATAAGCCCGTAATTG & 42 \\
\hline $38[34]$ & $31[41]$ & TTAAATTTAATCAGAAAAGCCCGTAAAAAGTAATG & 35 \\
\hline $34[125]$ & $32[119]$ & AAGAAAATAGCTATCTTACCGACTAACCCACAAGA & 35 \\
\hline $32[55]$ & $32[56]$ & AGGGTGACTGATAAATTAACACAACATATCACATTAATTGAA & 42 \\
\hline $35[87]$ & $32[70]$ & GTAGGTGCCTCCTCACAGTTTCCAGATGAGTGAGCTAAC & 39 \\
\hline $37[98]$ & $32[91]$ & TAGAAAATTATTACTCAGAGGAATAATAAGCTGGG & 35 \\
\hline $31[140]$ & $33[146]$ & CTACGAATAAACGGGTAAAATAATGAGGACTAAAG & 35 \\
\hline $36[34]$ & $33[48]$ & CTAGCATAGAGTCTGGAGCAATTTTGAGTTCAACCGTTCTAG & 42 \\
\hline $32[41]$ & $34[28]$ & CGGAGACAGTCAAATATGATAAGATCTA & 28 \\
\hline $34[90]$ & $34[91]$ & GAGTGAAATTGTTAGAAGCATAAAGTGTAAAGCAGGTTACCA & 42 \\
\hline $35[140]$ & $35[139]$ & CAGCAGCCTACAGAGGCTTGCCCTTTTTAAGATCGTCACCCT & 42 \\
\hline $33[147]$ & $35[150]$ & ACTTTTTCATGA??GAGGGTAGCAACGGGAAA & 32 \\
\hline $35[56]$ & $35[55]$ & GAATTCGCATGCCGGAGAGGGTAGCTATACAAGAGAATCGTC & 42 \\
\hline $39[140]$ & $36[133]$ & TTTCGAGTGCGCCGCTTTGACCAAAAGAATACACTAATGCCACCGCTTT & 49 \\
\hline $36[132]$ & $36[140]$ & TGCGGAGATACCATCGCCCACGCATAACTTAAAGG & 35 \\
\hline $32[118]$ & $37[111]$ & ATTGAGTATAGCAAGTAAGCAGATAGCCGCAATAAAGACTCCTACATAC & 49 \\
\hline $28[118]$ & $37[125]$ & CGTCAAACATAAAAACGCAAAATAAAGGTGGCAAC & 35 \\
\hline $32[48]$ & $37[55]$ & GAAAGGCTGTAGGTAAAGATTGCATGAACGGTAATCCAAAAACAGGACT & 49 \\
\hline $33[70]$ & $37[69]$ & CGAGCCGTCCGCTCACAATTCTAATCATTCCCCGGCGCGTGC & 42 \\
\hline $28[76]$ & $37[83]$ & GCAACAGGCGTATTTTTCACGCTGTTCTTCGCGTC & 35 \\
\hline
\end{tabular}




\begin{tabular}{|c|c|c|c|}
\hline $30[90]$ & $37[97]$ & CCAGCTGAGGGTGGTTTTTTTAGTTTATGCAAACG & 35 \\
\hline $26[125]$ & $39[118]$ & GAGCCTAATTTGCCCAATCCAAAGGGCGTATGGTT & 35 \\
\hline $28[139]$ & $39[139]$ & TACAACGCACTCATACAATGACAACAATGCTTATCAGCTTGC & 42 \\
\hline $35[151]$ & $39[150]$ & GACCAGGGAGCGATATACGATAGTGTGA & 28 \\
\hline $28[55]$ & $39[55]$ & AAATCGGTTCAACGTTAGATTGTATAAGTTGTTAAATCAGCG & 42 \\
\hline $43[140]$ & $40[133]$ & AACAACTGAACAACACGAGGCACCTGCTCCATGTTTTTGTATAATTGTA & 49 \\
\hline $24[125]$ & $41[118]$ & ATTAGTTGCTATTTCCAACGCCACCGACTTCATTA & 35 \\
\hline $26[55]$ & $41[55]$ & ATCCAATAGCATAATGCTCATTTTTTAATTTCATCAACATAG & 42 \\
\hline $26[90]$ & $41[97]$ & GTTTGCCTATAAATCAAAATTAATTAGAGGTAAAT & 35 \\
\hline $38[69]$ & $42[56]$ & GTCATACGCACTCTCAGCGCAGTTACCTAATCGTTAACGGCA & 42 \\
\hline $24[76]$ & $42[84]$ & TGAGTGTTCGGCAATTCAGCAGCAGCCAGCGGTGCCGGTGAAGCTACAC & 49 \\
\hline $24[139]$ & $43[139]$ & ACCAACTAGCCGGATAAAGGAATTGCTAGCGGGATTTTGCTA & 42 \\
\hline $39[151]$ & $43[150]$ & ATTCAAAAGGTTGAAAATAGAAAGTTCA & 28 \\
\hline $25[49]$ & $43[55]$ & GTAGCATTCTAAATGTGAGCGGGGATAGGTCACGG & 35 \\
\hline $26[111]$ & $44[112]$ & AGTTACAAATCTTATGCACCCGATGCGGGAGGTTTCACCAAT & 42 \\
\hline $47[140]$ & $44[133]$ & GTAACACTGTAGCATCTTGACGCTGGCTGACCTTCAAGAGGAAATTTTC & 49 \\
\hline $41[98]$ & $45[111]$ & ATTGACGGAAATTATTGAGCCAGCACCAGAAACCAATCAAGT & 42 \\
\hline $20[125]$ & $45[125]$ & CATTACCGCGCCCACGGTATTCGTTTTCTTGCCTTTAGCGTC & 42 \\
\hline $22[55]$ & $45[55]$ & TCCCAATAATAACCATGTTGGTGTAGATGCCTCAGGAAGAGC & 42 \\
\hline $20[76]$ & $45[83]$ & AGCTTAGCGCTATTGCATCAGCGCCGGGCGCGGTT & 35 \\
\hline $24[90]$ & $46[91]$ & GCCCGAGTGGACTCATAAATCTTTCCCTTAGAAATTCATAGC & 42 \\
\hline $47[98]$ & $46[98]$ & CGTTTGCTTTTCGG & 14 \\
\hline $20[139]$ & $47[139]$ & TAACAAAAGAGTAATTCCACAGACAGACACAACCCATGTACC & 42 \\
\hline $43[151]$ & $47[150]$ & ACAGACGTTAGATCTAACAACGCCTGAG & 28 \\
\hline $20[55]$ & $47[55]$ & TAGCTCATAACAGTGCTCGCACTCCAGCCATTCAGGCTGCGT & 42 \\
\hline $51[140]$ & $48[133]$ & GTATAGCTTAGTACAGATGGTCAGAACGAGTAGTACTCATTCAAGCCCA & 49 \\
\hline $43[56]$ & $48[63]$ & GTAAAGGTTTCTTTCCCTGCGCTCAATCCGGGGTCGCCATCCTGCCAAC & 49 \\
\hline $18[111]$ & $49[125]$ & CCAAGTAAATAATCGGCTGTCCACCAGATCAGAGCCACCACC & 42 \\
\hline $18[55]$ & $49[55]$ & TTAATTGATATAATCCGCAACTGTTGGGATGTGCTGCAAGCG & 42 \\
\hline $16[76]$ & $49[83]$ & AACTTTTGTAAATGCACATCCCAGCGTGGTGCTGG & 35 \\
\hline $18[104]$ & $50[91]$ & CCGCACTCAGAGAGACTACCTAAATCCAATCGCAACGCCGCC & 42 \\
\hline $16[139]$ & $51[139]$ & TTATGCGGGGCTTGCGCCACCCTCAGCATTGGGTTGATATAA & 42 \\
\hline $47[151]$ & $51[150]$ & TTTTTTTCAGGCCACCCAGGAGGTCCGG & 28 \\
\hline $18[48]$ & $51[55]$ & CTCCTTTCAGGATTAGAGAGTGCGCGATTAAGTTGAAGCTTTCAGAGAA & 49 \\
\hline $55[140]$ & $52[133]$ & ATGCCCCTGAAAGTCTAACGGGAAAAATCTACGTTTAAGAACGTGCCGT & 49 \\
\hline $12[118]$ & $53[125]$ & CAGTAATTAATTCTCTTTTGACCGTTCCAGTAAGC & 35 \\
\hline $49[56]$ & $53[69]$ & GACTTGTAGAACGTTCATAACCGTTTTTGATTGCCCGCACAG & 42 \\
\hline $12[76]$ & $53[83]$ & CAAATTCATCATAAACATCGAGCGGCCTTTAGTGA & 35 \\
\hline $14[90]$ & $53[97]$ & CCGACCGAAAAAGCCTGTTCCACTGGTAAAAGCGC & 35 \\
\hline $51[98]$ & $54[105]$ & GAGGTTGAGGCAGGTAAATCCGAATTTATGATACA & 35 \\
\hline $12[111]$ & $55[118]$ & AAGAGAAAACGCCAACATGTACCTCGTTTGAGTAA & 35 \\
\hline $10[125]$ & $55[139]$ & TCATAACATTTAGGCAGAGTTAGGAATATAAGAGCACAGTTA & 42 \\
\hline $51[151]$ & $55[150]$ & AATTACCAGGTTAGGATTGAAACACTGC & 28 \\
\hline
\end{tabular}




\begin{tabular}{|l|l|l|l|}
\hline $12[48]$ & $55[55]$ & TGCTTTAAAATATTCATTGAACAGAGGGGGATCAA & 35 \\
\hline
\end{tabular}

Table S3. Staple strands of DNA origami Arm

\begin{tabular}{|c|c|c|c|}
\hline Start & End & Sequences 5'-3' & Length \\
\hline $5[105]$ & $0[112]$ & GCCATTGAATATCCTACTTCTTCACGCACGGTACGCCAGAATGAGCCGT & 49 \\
\hline $6[153]$ & $0[140]$ & GTATTAACAGAAGATTAAAAACGCGAACAAATATCTTTAGGA & 42 \\
\hline $3[42]$ & $0[52]$ & GTTTGGAAAGGAGCTTTTGCGGAACAAAACTC & 32 \\
\hline $9[63]$ & $0[73]$ & AAAACAACAAACAATTCGACAGAGAGCGGGAGCTAAACAGGAGACT & 46 \\
\hline $5[21]$ & $3[34]$ & AAATAAATCAAAАTTTCATCAATATAAT & 28 \\
\hline $6[121]$ & $4[126]$ & GGAAAAAACGCTCATGGCAAACTA & 24 \\
\hline $6[37]$ & $4[42]$ & ATACGTAGATTTTCAGGGAAGGGT & 24 \\
\hline $4[118]$ & $5[104]$ & TGCTGGTCAACAGGTTATTTACATTGGCAGATTCCACCGCCA & 42 \\
\hline $2[118]$ & $6[122]$ & CTGTCCATTGATTATCTGACCGACCAGTAATAAAAAAAT & 39 \\
\hline $3[161]$ & $6[154]$ & AATATCACCAGCAGCAAATGAACAGTGCGCGGTCA & 35 \\
\hline $4[34]$ & $6[21]$ & TACCATAGAAATTGTACAGTAACAGTAC & 28 \\
\hline $6[48]$ & $6[49]$ & ACGTCAGGCCTGATTGCTTGGGGTCGAGTGGCGAGAAAGGTA & 42 \\
\hline $2[76]$ & $6[77]$ & GCTTTCCCTACAGGCCATCACAAAGCACTAAATCGGACGGGG & 42 \\
\hline $2[160]$ & $7[160]$ & AATATCTAACCTCAATCGCCATAAAACA & 28 \\
\hline $4[97]$ & $7[97]$ & TATAGCGGTCACGCCTATGGTTGCTTTTACCTTTTAAAGGGA & 42 \\
\hline $2[139]$ & $8[126]$ & GAGGCCATGCCTGACGCCAACAGAGATA & 28 \\
\hline $0[135]$ & $8[140]$ & TAAATTAGTCTTTAATGTACCGAA & 24 \\
\hline $2[55]$ & $8[42]$ & CCACCAGTTATACTTTTGAATACCAAGT & 28 \\
\hline $0[51]$ & $8[56]$ & GTAAAACAAACATCAAGAAGTTTT & 24 \\
\hline $4[76]$ & $8[63]$ & AACCACCAGCGGGCAAAGCCGGCGAACGGTGCCGTCCAAATC & 42 \\
\hline $5[77]$ & $8[84]$ & GCTAGGGCGCTGGCAAGTGTTCCGATTTAGAGCTTGAACCCTTTACACT & 49 \\
\hline $1[140]$ & $9[160]$ & CAAATCAACAGTTGAAAGGAATTATCTATGATAGC & 35 \\
\hline $2[97]$ & $9[97]$ & CGTGACGAGCACGTTAAAGGGATTTTAGTTTGAGGTCATTTG & 42 \\
\hline
\end{tabular}

Table S4. Staple strands of AuNR captures (arm)

\begin{tabular}{|l|l|l|r|}
\hline Start & End & Sequences ${ }^{\prime}$-3' & Length \\
\hline $9[126]$ & $2[119]$ & AATGGCTCAACTAATAGATTACCTGAGAAAAGAGT & 35 \\
\hline $3[140]$ & $2[140]$ & GTATCACCTTGCTGGGTCAGTTGGCAGT & 28 \\
\hline $9[42]$ & $2[35]$ & GATGATGTTAAATCCTTTGCCATTATCAGGAATTA & 35 \\
\hline $3[56]$ & $2[56]$ & TCCTTAATGCGCCGTCGTTAGAATCAAA & 28 \\
\hline $9[84]$ & $2[77]$ & AACAATTATTTAGAAGTATTAGGCCGATATAACGT & 35 \\
\hline $9[98]$ & $2[98]$ & AAAATACGTGGCACCAATAGATAATACAACAGGAAAATTAAC & 42 \\
\hline $9[112]$ & $4[119]$ & AGACAATATTTTTGGAACCCTGTAATAATCGGCCT & 35 \\
\hline $4[139]$ & $4[140]$ & AAGAACTAAATACCTACATCCGCCTGCAAAAATCTAAAGCAG & 42 \\
\hline $0[30]$ & $4[35]$ & CGTCCTGAGCAAAAGAATACAAAACCTGATTTAGAACC & 38 \\
\hline $4[55]$ & $4[56]$ & AATAATGTTAAGGGAAGAAAGCGAAAGGACACCCGCCGCGTG & 42 \\
\hline $0[72]$ & $4[77]$ & TTAAATTAATTACATTTACGTGAAGCGCGTATGCGCGT & 38 \\
\hline $7[98]$ & $4[98]$ & GCCACCAGTCACACTGAAAGCGTAAGTTGTAGCAAAGAACAA & 42 \\
\hline
\end{tabular}


Table S5. Staple strands of AuNR captures (plate)

\begin{tabular}{|l|l|l|r|}
\hline Start & End & Sequences $5^{\prime}-3^{\prime}$ & Length \\
\hline $30[76]$ & $35[86]$ & GAATCGGTGCCCGCTTGAGGAGGTCATAGCTGTTTCCT & 38 \\
\hline $32[90]$ & $35[97]$ & GTGCCTATCGGGAAACCTGAGGCAGTATGAAACCG & 35 \\
\hline $27[70]$ & $39[86]$ & CTGAGAGGACGATCGTGGTGCTGCGGCCAGA & 31 \\
\hline $28[90]$ & $39[97]$ & CACCAGTGCAAGCGGTCCAGCGAGGGAGTCACAAT & 35 \\
\hline $22[76]$ & $43[86]$ & GTGAGTGACTATTAACTGTTGGCTCGTCATAAACATCC & 38 \\
\hline $21[98]$ & $43[97]$ & ATAGAAGAACCGTCTATCAACCAACGTCCAGCACCGCAAAAT & 42 \\
\hline $18[76]$ & $47[86]$ & CTCCGGCAGTGAATGCAAGAACACGCAACCAGCTTACG & 38 \\
\hline $20[90]$ & $47[97]$ & GAAAACAAATCATAGGTCTTCCCTCAGATTATTAG & 35 \\
\hline $14[76]$ & $51[86]$ & AATAAGGTCTTCTGCGATGCTTCGTCTCGTCGCTGGCA & 38 \\
\hline $16[90]$ & $51[97]$ & AAAGAACTTTAATGGTTTGCCAGCCAGATTGACAG & 35 \\
\hline $11[63]$ & $55[86]$ & ACGCTCAACAGTAGCTTTTGCTCATTTGCCGCCAGCAG & 38 \\
\hline $12[90]$ & $55[97]$ & TCATATGTTGAGAATCGCCATAAAAACCGTTTTAA & 35 \\
\hline
\end{tabular}

Table S6. Sequences of SH-DNA on AuNRs

\begin{tabular}{|c|c|}
\hline Name & Sequences 5'-3' \\
\hline SH-DNA-T & HS-TTT TTT TTT TTTT \\
\hline SH-DNA-b & HS-TTT TGA GTT AGT \\
\hline SH-DNA-c & HS-TTT CAG TGA TGT \\
\hline
\end{tabular}

Table S7. Switching systems on DNA origami templates

\begin{tabular}{|c|c|c|c|}
\hline \multicolumn{4}{|c|}{ Chiral Center 1} \\
\hline Start & End & Sequences 5'-3' & Name \\
\hline $2[34]$ & $0[31]$ & TCATCATATTCCTGAAAGTTTGAGTAACCGAA TTTTTT TGTAGTTG & $1 \mathrm{R}-0$ \\
\hline $6[142]$ & $6[143]$ & $\begin{array}{l}\text { TGACGCTCAATCGTCTGGGGACATTCTGGAACCACCAGCATT TTTTTT } \\
\text { TGTAGTTG }\end{array}$ & $1 \mathrm{R}-6$ \\
\hline $14[55]$ & $53[55]$ & $\begin{array}{l}\text { CCAATACAACTACA TTTTTT } \\
\text { ATTATAGTCAAATACAGTGGAGCCGCCATTACCAGTCCCGAA }\end{array}$ & $1 \mathrm{R}-14$ \\
\hline \multirow[t]{3}{*}{$30[118]$} & $35[125]$ & $\begin{array}{l}\text { CCAATACAACTACA TTTTTT } \\
\text { AATTAACAGCGCTAATGATTATAACGGAATACCCA }\end{array}$ & $1 \mathrm{R}-30$ \\
\hline & & TGTAGTTGTATTGGTGAAGT & 1R-Blocker \\
\hline & & ACTTCACCAATACAACTACA & 1R-Activator \\
\hline $3[126]$ & $0[136]$ & CATCACTCCGAGTAAGTGTTTTTATAATGCAC TTTTTT TACTAC TC & $1 \mathrm{~L}-0$ \\
\hline $8[34]$ & $6[38]$ & TCGCGCAACAATAACGGATTCATGA TTTTTT TACTACTC & $1 \mathrm{~L}-6$ \\
\hline $30[55]$ & $26[42]$ & $\begin{array}{l}\text { TCTTGTGAGTAGTA TTTTTT } \\
\text { GATAAAAATTTTTACCTTTATTTGTACCGCCTCAGAAATCAT }\end{array}$ & $1 \mathrm{~L}-30$ \\
\hline \multirow[t]{3}{*}{$14[118]$} & $51[125]$ & $\begin{array}{l}\text { TCTTGTGAGTAGTA TTTTTT } \\
\text { ATGTTCAAGATAAGAAACAAATCAGACGATTGGCC }\end{array}$ & $1 \mathrm{~L}-14$ \\
\hline & & TACTACTCACAAGATGAAGA & 1L-Blocker \\
\hline & & TCTTCATCTTGTGAGTAGTA & 1L-Activator \\
\hline \multicolumn{4}{|c|}{ Chiral Center 2} \\
\hline
\end{tabular}




\begin{tabular}{|c|c|c|c|}
\hline $2[34]$ & $0[31]$ & TCATCATATTCCTGAAAGTTTGAGTAACCGAA TTTTTT ACACTCTA & $2 \mathrm{R}-0$ \\
\hline $6[142]$ & $6[143]$ & $\begin{array}{l}\text { TGACGCTCAATCGTCTGGGGACATTCTGGAACCACCAGCATT TTTTTT } \\
\text { ACACTCTA }\end{array}$ & $2 \mathrm{R}-6$ \\
\hline $14[55]$ & $53[55]$ & $\begin{array}{l}\text { CAGGAGTAGAGTGT TTTTTT } \\
\text { ATTATAGTCAAATACAGTGGAGCCGCCATTACCAGTCCCGAA }\end{array}$ & $2 \mathrm{R}-14$ \\
\hline \multirow[t]{3}{*}{$30[118]$} & $35[125]$ & $\begin{array}{l}\text { CAGGAGTAGAGTGT TTTTTT } \\
\text { AATTAACAGCGCTAATGATTATAACGGAATACCCA }\end{array}$ & $2 \mathrm{R}-30$ \\
\hline & & ACA CTC TA CTC CTG TCC TCC & 2R_BLOCKER \\
\hline & & GGA GGA CAG GAG TAG AGT GT & 2R-ACTIVATOR \\
\hline $3[126]$ & $0[136]$ & CATCACTCCGAGTAAGTGTTTTTATAATGCAC TTTTTT GTT AGTTG & $2 \mathrm{~L}-0$ \\
\hline $8[34]$ & $6[38]$ & TCGCGCAACAATAACGGATTCATGA TTTTTT GTTAGTTG & $2 \mathrm{~L}-6$ \\
\hline $30[55]$ & $26[42]$ & $\begin{array}{l}\text { CACACACAACTAAC TTTTTT } \\
\text { GATAAAAATTTTTACCTTTATTTGTACCGCCTCAGAAATCAT }\end{array}$ & $2 \mathrm{~L}-30$ \\
\hline \multirow[t]{3}{*}{$14[118]$} & $51[125]$ & $\begin{array}{l}\text { CACACACAACTAAC TTTTTT } \\
\text { ATGTTCAAGATAAGAAACAAATCAGACGATTGGCC }\end{array}$ & $2 \mathrm{~L}-14$ \\
\hline & & GTT AGT TGTGT GTG GTG TTG & 2L_BLOCKER \\
\hline & & CAA CAC CAC ACA CAA CTA AC & 2L-ACTIVATOR \\
\hline \multicolumn{4}{|c|}{ Chiral Center 3} \\
\hline $2[34]$ & $0[31]$ & TCATCATATTCCTGAAAGTTTGAGTAACCGAA TTTTTTACTGGTGA & $3 \mathrm{R}-0$ \\
\hline $6[142]$ & $6[143]$ & $\begin{array}{l}\text { TGACGCTCAATCGTCTGGGGACATTCTGGAACCACCAGCA } \\
\text { TTTTTTTTACTGGTGA }\end{array}$ & $3 \mathrm{R}-6$ \\
\hline $14[55]$ & $53[55]$ & $\begin{array}{l}\text { TATGTATCACCAGTTTTTTT } \\
\text { ATTATAGTCAAATACAGTGGAGCCGCCATTACCAGTCCC }\end{array}$ & $3 \mathrm{R}-14$ \\
\hline \multirow[t]{3}{*}{$30[118]$} & $35[125]$ & $\begin{array}{l}\text { TATGTATCACCAGTTTTTTT } \\
\text { AATTAACAGCGCTAATGATTATAACGGAATACCCA }\end{array}$ & $3 \mathrm{R}-30$ \\
\hline & & ACTGGTGATACATAGTGTTG & 3R_BLOCKER \\
\hline & & CAACACTATGTATCACCAGT & 3R-ACTIVATOR \\
\hline $3[126]$ & $0[136]$ & CATCACTCCGAGTAAGTGTTTTTATAATGCAC TTTTTTCTCTAACA & $3 \mathrm{~L}-0$ \\
\hline $8[34]$ & $6[38]$ & TCGCGCAACAATAACGGATTCATGA TTTTTTCTCTAACA & $3 \mathrm{~L}-6$ \\
\hline $30[55]$ & $26[42]$ & $\begin{array}{l}\text { TACAAGTGTTAGAGTTTTTT } \\
\text { GATAAAAATTTTTACCTTTATTTGTACCGCCTCAGAAAT }\end{array}$ & $3 \mathrm{~L}-30$ \\
\hline \multirow[t]{3}{*}{$14[118]$} & $51[125]$ & $\begin{array}{l}\text { TACAAGTGTTAGAGTTTTTT } \\
\text { ATGTTCAAGATAAGAAACAAATCAGACGATTGGCC }\end{array}$ & $3 \mathrm{~L}-14$ \\
\hline & & CTCTAACACTTGTAAGTCTG & 3L_BLOCKER \\
\hline & & CAGACTTACAAGTGTTAGAG & 3L-ACTIVATOR \\
\hline
\end{tabular}

